\title{
Localisation of plastic deformation in stretching plates: microstructure effects
}

\author{
J.L. Dequiedt ${ }^{* \dagger}$ C. Denoual ${ }^{* \dagger}$ \\ *CEA, DAM, DIF, F-91297 Arpajon, France. \\ ${ }^{\dagger}$ Université Paris-Saclay, CEA, LMCE, F-91680 Bruyères-le-Chatel, France. \\ e-mail: jean-lin.dequiedt@cea.fr, christophe.denoual@cea.fr
}

\begin{abstract}
The localization of plastic deformation in dynamically expanding shells of ductile metals is usually investigated at the macroscopic scale through the analysis of instable perturbation modes of the structure (among the numerous works on this topic, let us mention [1]). Still, at the scale of the microstructure, i.e. the one of the crystal aggregate, the deformation is inherently heterogeneous from grain to grain since the beginning of loading. Simulations of stretching plates including the polycrystalline structure of the material, by means of a crystal plasticity FE code, give a way to investigate the connection between grain scale strain heterogeneity and macroscopic localization. Crystal plasticity is ruled by a Teodosiu type constitutive model [2] which integrates the high anisotropy of slip system interactions, identified by dislocation dynamics simulations.
\end{abstract}

At the beginning of loading, strain organizes at the scale of a few grains and progressively larger strain patterns arise as if the following stages were controlled by structural perturbation modes: however, marks of the initial strain distribution are still visible (fig. 1). At last, a few of these zones strongly concentrate deformation and unloading is observed in the rest of the plate; this ultimate stage seems to be driven by what resembles a "Mott type" obscuration [3].

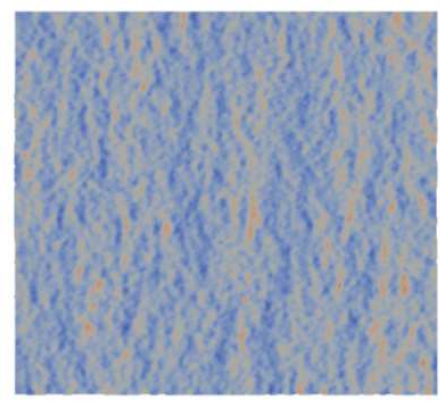

$t=20 \mu \mathrm{s}$

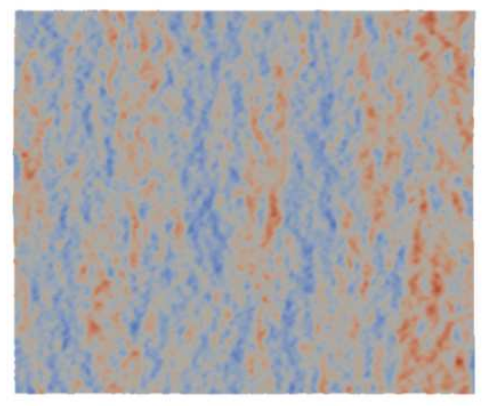

$t=40 \mu \mathrm{s}$

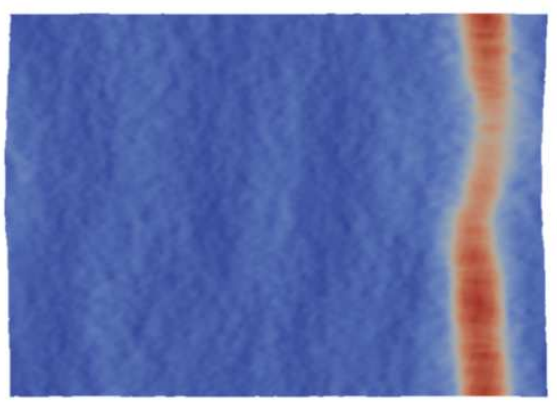

$t=80 \mu \mathrm{s}$

Fig. 1. Strain maps in plane strain stretching at different times.

The importance of these different stages depends on the loading conditions and microstructural characteristics. When increasing the loading rate, the last stage is delayed and structural modes have more time to develop. On the contrary, a compression texture, constituted of strong fibers, yields a higher initial strain heterogeneity of which the final localization patterns keeps memory.

\section{REFERENCES}

[1] M. Xavier, C. Czarnota, D. Jouve, S. Mercier, J.L. Dequiedt, and A. Molinari, "Extension of linear stability analysis for the dynamic stretching of plates : spatio-temporal evolution of the perturbation". Eur. J. Mech. A Solids, 79, 1-19 (2020).

[2] C. Teodosiu, J.L. Raphanel and L. Tabourot, "Finite element simulation of the large elastoplastic deformation", In: Large Plastic Deformations edited by C. Teodosiu , J.L. Raphanel and F. Sidoroff, Rotterdam, A.A. Balkema, pp. 153-168 (1991).

[3] J.L. Dequiedt and C. Denoual, "Localization of plastic deformation in stretching sheets with a crystal plasticity approach: competition between weakest link and instable mode controlled process", Int. J. Solids Struct., 210-211, 183-202 (2021). 